\title{
APLICAÇÃO DO SOFTWARE COMSOL NA ANÁLISE DE LIMITAÇŌES DIFUSIONAIS INTERNAS DE CALOR E MASSA EM CATALISADORES POROSOS COM FORMAS GEOMÉTRICAS NÃO CONVENCIONAIS
}

\author{
Rosenir Rita de Cassia Moreira da Silva ${ }^{1}$ \\ Arlindo de Almeida Rochal \\ Priscila Dias Sily ${ }^{1}$ \\ Fabiana Vassalo Caldas ${ }^{1}$
}

\begin{abstract}
Resumo: O objetivo deste trabalho é apresentar a aplicação de softwares, especialmente o COMSOL, em problemas envolvendo cálculos complexos, em particular, os relacionados às limitaçóes difusionais internas à transferência de massa e calor, simultâneos, que ocorrem em reações químicas catalíticas usando, como catalisadores, sólidos porosos com diferentes geometrias. Um exemplo de aplicação é apresentado utilizando-se catalisadores comerciais, de diversas geometrias, empregados no processo industrial de reforma a vapor do metano. O problema é formulado a partir das equações gerais da conservação de massa e energia, disponíveis no COMSOL e resolvido utilizando os métodos numéricos presentes no software. As soluções são apresentadas em forma de gráficos uni, bi e tridimensionais permitindo-se uma boa visualização dos gradientes de concentração e temperatura no interior dos grãos catalíticos. Foram também determinados os fatores de efetividade para diversas geometrias dos grãos e os resultados comparados com as soluções existentes para grãos em forma de cilindro infinito através de rotinas desenvolvidas no software MAPLE.
\end{abstract}

Palavras-chave: Software COMSOL; Reação catalítica heterogênea; Limitações difusionais; Reforma a vapor do metano.

Abstract: The aim of this paper is to present the application of softwares, more specifically COMSOL, in problems related to internal difusional limitations associated with simultaneous heat and mass transfer in catalytic chemical reactions in porous catalysts with different unusual geometric forms. An example of application is presented by using commercial catalysts, with different geometric forms, employed in the industrial process of steam methane reforming. The problem is formulated starting from the basic general microscopic equations of mass and energy conservation using the software COMSOL, resulting in non-linear partial differential equations which are difficult to solve. The problems generated were solved numerically using COMSOL and the solutions are presented in the form of one, two and three-dimensional graphics showing the concentration and temperature profiles inside of the catalytic particles. The effectiveness factors of the catalytic particles for several unusual geometric forms of the particles were determined and the results were compared with the available solutions for particles with simpler geometric forms, by using specific procedures developed for the software MAPLE.

Keywords: COMSOL software; Heterogeneous catalytic reaction; Difusional limitations; Steam methane reforming

\footnotetext{
1 Universidade Federal Fluminense - Departamento de Engenharia Química e de Petróleo. Rua Passo da Pátria, 156, bl. D, sala 307, São Domingos, Niterói, RJ, Brasil. CEP. 24210-240. Email: rosenir@vm.uff.br
} 


\section{INTRODUÇÃO}

Nos últimos 50 anos ocorreu a revolução tecnológica da informática e da computação. Por meio de softwares especializados é possível, atualmente, estudar com mais facilidade problemas complexos de engenharia, de difícil solução. Esta constatação vem se mostrando uma realidade, permitindo que se façam simulações e modelagens de sistemas em que as análises experimentais não são suficientes para o entendimento dos fenômenos envolvidos. A utilização dessas ferramentas computacionais, em particular dos softwares CFD, permite a visualização do comportamento dos fluidos em diversas situações, envolvendo conhecimentos de fenômenos de transporte, termodinâmica, sistemas de reações químicas, além de outros, correspondendo a um ponto fundamental na predição de variáveis importantes para a elaboração de um projeto e também no processo de ensino-aprendizagem, em que funciona como um laboratório virtual. Problemas relativos aos mecanismos de transferência de massa, calor e quantidade de movimento, associados às reaçôes químicas catalíticas, como os que serão abordados neste trabalho, geralmente apresentam um grau de dificuldade considerável, que podem ser mais bem compreendidos com o uso desses softwares.

$\mathrm{Na}$ maioria dos livros, métodos simplificados são propostos para a solução de tais problemas. Esses métodos, em muitos casos, somente se aplicam a determinadas situações e dependem da utilização de gráficos, os quais implicam em soluções aproximadas e imprecisas (FIGUEIREDO e RIBEIRO, 1989; FOGLER, 2002; FROMENT e BISCHOFF, 1979; SATTERFIELD, 1991). Porém, utilizando como ferramenta softwares de última geração, pode-se obter soluções exatas e resultados precisos de forma mais rápida e simples.

Dentre os softwares disponíveis atualmente destacam-se o MAPLE e o COMSOL, que serão utilizados no presente trabalho. $\mathrm{O}$ emprego desses softwares possibilita a formulação dos problemas de fenômenos de transporte partindo dos princípios básicos, bem como, a solução, que no caso do MAPLE pode ser feita algebricamente e numericamente, e no caso do COMSOL, um software CFD, numericamente. Ambos proporcionam um ambiente computacional interativo, reduzindo o esforço necessário em manipulações algébricas longas e tediosas e a necessidade de consultar tex- tos sobre métodos matemáticos numéricos para a solução dos problemas. Como conseqüência direta, o usuário fica mais livre para verificar e expandir as concepções intelectuais, as hipóteses e as suposições adotadas nos modelos a serem estudados e para estimular a sua criatividade no desenvolvimento de modelos mais apropriados para a situação física desejada. Para fins acadêmicos, abre uma nova perspectiva para o estudante, futuro profissional, pois não fica limitado a problemas simplificados com geometrias tradicionais, podendo usar formas geométricas diferentes, como as usadas industrialmente. $\mathrm{O}$ tratamento não fica mais restrito a sistemas unidimensionais e lineares, podendo ser tratado de forma tridimensional com equações diferenciais não lineares.

Recentes trabalhos têm sido desenvolvidos no curso de Engenharia Química da UFF usando extensivamente o MAPLE, não somente em problemas de fenômenos de transporte, mas em sistemas reacionais heterogêneos, usando-se catalisadores em formas esféricas, cilíndricas e de placas planas (CALDAS et al., 2005 2009; SILY et al., 2006). Considerando-se apenas uma variável geométrica, esses sistemas originam equaçôes diferenciais ordinárias, facilmente resolvidas pelo MAPLE. Particularmente, o ensino torna-se mais interessante à medida que o aluno pode observar os efeitos dos diversos parâmetros reacionais nas limitações difusionais internas e externas. No entanto, algumas dificuldades surgem ao se tratar problemas com geometrias diferentes, pois o MAPLE tem limitação no tocante à solução analítica e numérica das equaçôes diferenciais parciais resultantes de sistemas bi ou tridimensionais. Com a introdução do COMSOL, um software CFD, uma nova perspectiva se abre, permitindo desenvolver, formular e obter soluções numéricas de problemas de química e de engenharia química, tanto fundamentais, como aplicados, de grande complexidade.

O presente trabalho tem como objetivo apresentar um exemplo de aplicação do software COMSOL em reaçôes catalíticas empregando-se catalisadores com diferentes formas geométricas. A Figura 1 apresenta alguns desses catalisadores comerciais, usados industrialmente nos processos de reforma a vapor, contendo diversos furos internos para reduzir as limitaçōes difusionais. Usou-se também o caso do cilindro infinito para se comparar as soluções encontradas no COMSOL com 
as do MAPLE para sistemas unidimensionais. As soluções são apresentadas em formas de gráficos $2 \mathrm{D}$ e $3 \mathrm{D}$, permitindo-se uma boa visualização dos gradientes de concentração e temperatura no interior dos grãos catalíticos, expressos por meio de variaçóes nas tonalidades com suas respectivas escalas de grandeza.

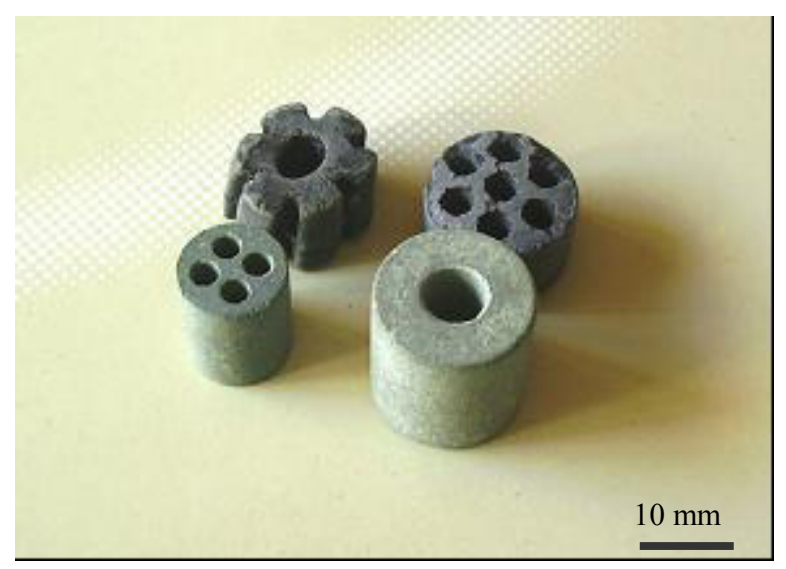

Figura 1 - Formas geométricas de catalisadores empregados na Reforma a Vapor do Metano

\section{CATALISADORES USADOS NA REFORMA A VAPOR}

A reforma a vapor do metano é uma das reações mais importantes industrialmente para produção do gás de síntese e, também, uma das rotas mais utilizadas para a geração de hidrogênio. O catalisador é o níquel suportado em alumina, podendo conter promotores (TWIGG, 1980). A reação é fortemente endotérmica e ocorre a altas temperaturas, na faixa de $700-800^{\circ} \mathrm{C}$, podendo ser representada da seguinte forma:

$$
\mathrm{CH}_{4}+\mathrm{H}_{2} \mathrm{O} \rightarrow \mathrm{CO}+3 \mathrm{H}_{2} \quad \Delta \mathrm{H}_{298}^{0}=206 \mathrm{~kJ} / \mathrm{mol}
$$

Cineticamente, inúmeros resultados de estudos da reação de reforma a vapor indicam que a reação é de primeira ordem em relação ao metano, mas não existe acordo com relação a outros parâmetros cinéticos, tais com as energias de ativação, por exemplo. Wei e Iglesia (2004) tem reportado valores da ordem de $102 \mathrm{~kJ} / \mathrm{mol}$ para energia de ativação desta reação. Em parte, isto é devido ao uso de diferentes catalisadores e condições experimentais, mas freqüentemente resulta da falta de uma análise mais aprimorada das limitaçôes difusionais internas à transferência de massa e de calor. Com os tamanhos de partículas de catalisadores relativamente grandes usados industrialmente nos reformadores a vapor, estes efeitos resultam em fatores de efetividade muito baixos (NIJEMEISLAND et al., 2004). Tem sido verificados, dependendo das condiçôes, fatores de efetividade para o catalisador da ordem de 0,3 na região de entrada e talvez da ordem de 0,01 na região de saída dos reformadores. Devido a isto, a atividade aparente aumenta com o decréscimo do tamanho do catalisador por diminuir as limitações difusionais, mas em contrapartida um aumento na queda de pressão ao longo do reformador restringe o tamanho do catalisador aos empregados industrialmente.

No caso de catalisadores na forma de cilindros com um ou mais furos internos, são observados tamanhos da ordem de $17 \mathrm{~mm}$ de diâmetro por $17 \mathrm{~mm}$ ou $10 \mathrm{~mm}$ ou até de $6 \mathrm{~mm}$ de comprimento. A atividade catalítica geralmente é proporcional à área da superfície externa. Tanto quanto possível, os catalisadores de $\mathrm{Ni}$ suportados, devem ter uma superfície de $\mathrm{Ni}$ ativo acessível ao gás reagente e ao mesmo tempo possuir uma boa resistência mecânica, o suficiente para resistir à abrasão e quebra durante o manuseio ou durante qualquer ciclo térmico que possa ocorrer.

As vazões do gás devem ser altas para evitar as limitações à transferência de massa externa e permitir uma boa troca térmica entre a parede do tubo e o catalisador. Várias formas têm sido empregadas, tais como, grãos cilíndricos, esféricos, em formas de anéis, tubos, barras e varas de diferentes tamanhos e diferentes seções transversais, com vários furos internos. Algumas dessas formas geométricas são apresentadas na Figura 1. A utilização de softwares, que permitam avaliar as limitações difusionais e simular situações e geometrias que possam diminuir estes efeitos, pode diminuir os esforços experimentais, gerando um conjunto de possibilidades mais adequadas para posterior teste catalítico.

\section{OS SOFTWARES MAPLE® E COMSOL®}

Os softwares MAPLE (ABELL, 1999; HECK, 1993; MEADE et al., 1996; CALDAS et al., 2005, 2009) e COMSOL (COMSOL, 2005) são complementares por possibilitarem a formulação dos problemas de fenômenos de transporte com ou sem reação química, partindo dos princípios básicos, e a solução algébrica ou numérica desses problemas, em um ambiente computacio- 
nal integrado e interativo. Reduz o esforço em manipulações algébricas e a necessidade de consultar textos sobre métodos matemáticos e numéricos necessários.

O MAPLE difere da maioria dos softwares usados atualmente devido à sua capacidade de realizar manipulação algébrica de expressões que envolvem símbolos, variáveis e operações de álgebra simbólica. Dessa forma, o software pode ser usado para formular e solucionar problemas obtendo soluções analíticas em diversas áreas do conhecimento. Além disso, o MAPLE também dispõe de um conjunto bastante completo de algoritmos para a solução numérica de problemas envolvendo equações diferenciais ordinárias. Pode ser programado de forma relativamente simples possibilitando a criação de rotinas ("procedures") adaptadas aos interesses específicos dos usuários, como as usadas para gerar as equaçóes de conservação microscópicas, e introduzir as simplificaçôes e as condiçôes de contorno e inicial.

Já o COMSOL é um ambiente interativo para desenvolvimento e simulação de modelos baseados em equações diferenciais parciais, que pode ser usado também para modelos baseados em equações diferenciais ordinárias. No modo multifísica, o COMSOL pode ser utilizado para modelar situações envolvendo transferência simultânea de calor, massa e quantidade de movimento (SILY et al., 2006). A utilização do COMSOL em princípio não exige qualquer programação ou desenvolvimento de algoritmos. Através de uma interface gráfica define-se a geometria do domínio, como mostra a Figura $2 \mathrm{a}$, selecionam-se as equações de conservação a serem utilizadas e as condições de contorno e iniciais. Em seguida, faz-se a geração automática da malha dos elementos finitos, como mostra a Figura $2 b$, e seleciona-se o método numérico de solução a ser utilizado na simulação do problema. O COMSOL dispõe ainda de uma grande capacidade de pós-processamento para análise e validação dos resultados. $\mathrm{O}$ arquivo da solução gerada nos elementos finitos pode ainda ser exportado para o MAPLE para análise aprofundada dos resultados gerados. Nesse contexto, o COMSOL pode ser encarado como um laboratório virtual para realização de experimentos numéricos de problemas físicos.

Problemas bi e tridimensionais estacionários ou transientes são formulados em termos de equações diferenciais parciais gerando problemas de valor de contorno e inicial cujas soluções são mais complexas. No caso de problemas lineares, é possível obter as soluções usando o método de separação de variáveis e séries de Fourier. Para obter essas soluções usando o MAPLE têm sido desenvolvidas as rotinas específicas para esse fim. No caso de problemas de valor inicial, em domínios finitos, semi-infinitos, com duas variáveis independentes, a solução pode ser obtida usando o método da transformada de Laplace no tempo e obtenção da solução da equação diferencial ordinária no espaço e, posteriormente, a inversão da solução final. Para o caso de problemas não-lineares, em que os métodos numéricos são a única opção para a solução, preferência deve ser dada ao COMSOL que obtém a solução pelo método dos elementos finitos. Nesse caso, o problema pode ser formulado diretamente no COMSOL para obtenção da solução numérica desejada. Com a utilização do MAPLE e COMSOL os estudantes podem obter uma boa formação em química e engenharia química sem necessariamente conhecer todos os detalhes dos métodos matemáticos e numéricos necessários para formular e solucionar os problemas.

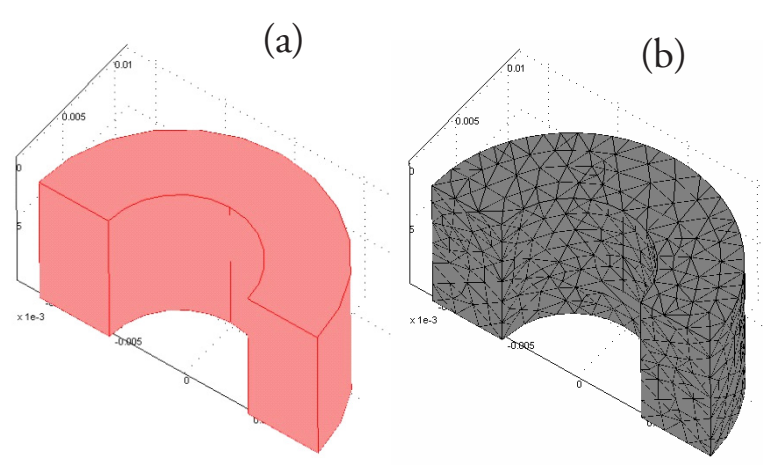

Figura 2 - (a) Geometria do domínio definida pela interface gráfica de um catalisador em forma de cilindro vazado e (b) Geometria contendo a malha dos elementos finitos gerada

\section{FORMULAÇÃO DO PROBLEMA}

O problema a ser estudado considera a reação química catalítica irreversível $\mathrm{A} \rightarrow \mathrm{B}$, cuja taxa de reação $\left(-r_{A}\right)$ é de primeira ordem em relação à concentração de $\mathrm{A}\left(C_{A}\right)$, ocorrendo num grão de catalisador, sujeito às limitaçôes difusionais internas de calor e massa. Os grãos possuem diferentes geometrias, como podem ser vistos na Figura 1. No presente trabalho, serão avaliados 
os perfis de concentração e de temperatura e os fatores de efetividade em grãos cilíndricos maciço e vazados com 1 (um), 4 (quatro) e 7 (sete) furos, tais como os empregados na reforma a vapor do metano.

Partindo-se das equações de conservação de massa e de energia, em regime permanente, obtém-se, respectivamente, as equações (1) e (2) (CREMASCO, 2004; INCROPERA et al., 2004).

$$
\begin{aligned}
& \operatorname{div}\left[D_{A e f} \operatorname{grad}\left(C_{A}\right)\right]=-r_{A}(1) \\
& \operatorname{div}[\lambda \operatorname{grad}(T)]=-(\Delta H) r_{A}
\end{aligned}
$$

Sendo $\left(-r_{A}\right)=k C_{A}$, onde $k$ é a constante de Arrhenius que depende da temperatura e pode ser descrita como $k=k_{0} \exp (-E / R T)$. Desta forma, verifica-se que a taxa de reação $\left(-r_{A}\right)$ é uma função fortemente dependente da concentração e da temperatura. $D_{A e f}$ e $\lambda$ são, respectivamente, a difusividade efetiva do reagente A através dos poros do grão catalítico e a condutividade térmica do grão.

As condições de contorno dependem da geometria. Para os grãos vazados, nas regiões que definem as superfícies internas dos furos e externas do grão, $C_{A}=C_{A s}$ e $T=T_{s}$, sendo $C_{A s}$ e $T$, respectivamente, a concentração de $\mathrm{A}$ e a temperatura na superfície. Nas faces criadas pelo plano que corta o cilindro no meio, axialmente, pode-se dizer que não há fluxo, devido à simetria radial.

Considerando o sistema de coordenadas apropriado, com fluxos radiais e axiais, obtêm-se as equações diferenciais. Essas equações também podem ser obtidas usando-se uma rotina desenvolvida no MAPLE (CALDAS et al., 2005, 2009), contendo a análise microscópica que engloba as equações da conservação da massa e da energia, que são apresentadas em suas formas gerais, para diversos sistemas de coordenadas, e depois transportadas para o COMSOL. De outro modo, pode-se também a partir do desenho feito em $2 \mathrm{D}$ ou $3 \mathrm{D}$, na interface gráfica definida pelo COMSOL, trabalhar com coordenadas retangulares ou cilíndricas.

Usando-se o COMSOL, o problema em particular pode ser tratado bidimensionalmente, nos casos dos cilindros maciços e vazados com apenas um furo, supondo cilindro com simetria axial, e, tridimensionalmente, para ambos os casos acima, acrescidos dos cilindros com quatro furos e com sete furos. A vantagem das soluções 3D reside na melhor visualização dos gradientes de concentração e de temperatura, embora muitas vezes envolva um maior esforço computacional.

Para permitir comparação entre os resultados obtidos com os diversos catalisadores, utilizou-se os mesmos parâmetros definidos por e Maymo e Smith (1966) e Weisz e Hicks (1962) para o cilindro fechado, a partir da adimensionalização das equações, sendo $\varphi$, o módulo de Thiele, e os parâmetros $\beta$ e $\gamma$, todos adimensionais, representados pelas equaçóes (3), (4) e (5).

$$
\begin{aligned}
& \phi=\frac{V_{\text {grão }}}{S_{\text {grão }}} \sqrt{\frac{k}{D_{A, e f}}} \\
& \beta=\frac{(-\Delta H) D_{A, e f} C_{A s}}{\lambda T_{s}} \\
& \gamma=\frac{E}{R T_{s}}
\end{aligned}
$$

Sendo $V_{\text {grãa }}$ o volume geométrico do grão, $S_{\text {grăo }}$ a área da superfície externa do grão, $\Delta H$, a entalpia da reação e $E$, a energia de ativação da reação. Cabe destacar que os valores negativos de $\beta$ representam reações endotérmicas e os valores positivos, reações exotérmicas.

Para os grãos contendo furos, $V_{\text {grão }}$ e $S_{\text {grão }}$ podem ser calculados da seguinte forma pelas equações (6) e (7).

$$
\begin{aligned}
& V_{\text {grão }}=\pi L\left(R^{2}-N R_{o}^{2}\right) \\
& S_{\text {grão }}=2 \pi\left(R L+N R_{O} L+R^{2}-N R_{o}^{2}\right)
\end{aligned}
$$

Sendo $R$ o raio externo do grão, $R_{o}$ o raio interno, $L$ o comprimento e $N$ o número de furos do grão.

Pode-se também calcular o fator de efetividade $(\eta)$, definido como a integral no volume, de acordo com a equação (8). O fator de efetividade é muito importante para se avaliar a importância dos problemas de difusão interna e expressa a razão entre a taxa real (observada) sujeita às limitaçōes difusionais internas e a taxa intrínseca 
da reação (sem limitação). Nas reações catalíticas em grãos isotérmicos, que não sofrem limitações à transferência de calor, que apresentam apenas problemas difusivos, o fator de efetividade varia entre zero a um. Nas reações, em que as limitações à transferência de calor são importantes, os fatores de efetividade podem ser maiores do que um, para as reações exotérmicas, isto é, para $\beta>1$. $\mathrm{O}$ COMSOL calcula numericamente os fatores de efetividade para qualquer geometria.

$$
\eta=\frac{\int\left(-r_{A}\right) d V}{\left(-r_{A}\right)_{S} V_{\text {grão }}}
$$

Os fatores de efetividade também podem ser calculados através do MAPLE, analiticamente, para sistemas unidimensionais.

No caso do MAPLE, inicialmente a análise microscópica engloba as equações da conservação de energia e da massa, que são apresentadas em suas formas gerais para diversos sistemas de coordenadas. Em regime permanente e considerando-se que os fluxos radiais predominam, obtém-se para um catalisador esférico, por exemplo, as seguintes equaçóes diferenciais:

$$
\begin{aligned}
& \frac{D_{A, \text { ef }}}{2} \frac{d}{d r}\left(r^{2} \frac{d C_{A}}{d r}\right)-r_{A}=0 \\
& \frac{\lambda}{r^{2}} \frac{d}{d r}\left(r^{2} \frac{d T}{d r}\right)+\left(-\Delta H_{R}\right)\left(r_{A}\right)=0
\end{aligned}
$$

Em seguida, efetuam-se as simplificações necessárias, bem como a adimensionalização do problema (MAYMO e SMITH, 1966; WEISZ e HICKS, 1962). Resolve-se o sistema de equações, no presente caso, uma diferencial (equação 11) e outra algébrica (equação 12).

$$
\begin{aligned}
& \frac{d^{2} y}{d x^{2}}+\frac{2}{x} \frac{d y}{d x}=\phi^{2} y \exp \left(\frac{\gamma \beta(1-y)}{1+\beta(1-y)}\right) \\
& T-T_{s}=\frac{\left(-\Delta H_{r}\right) D_{A, \text { ef }}}{\lambda}\left(C_{A s}-C_{A}\right)
\end{aligned}
$$

Sendo $y=\frac{r}{R}$ e T a temperatura.

\section{RESULTADOS OBTIDOS}

\subsection{RESULTADOS EM GRÁFICOS 2D}

\section{Catalisador em Forma de Cilindro Infinito:}

O problema do catalisador na forma de cilindro infinito pode ser tratado unidimensionalmente, pois os fluxos radiais predominam gerando equaçōes diferenciais ordinárias, facilmente resolvidas pelo MAPLE e COMSOL. A Figura 3 apresenta os resultados na forma de um gráfico $2 \mathrm{D}$, obtido no COMSOL, ilustrado com diversas tonalidades que permite verificar o gradiente de concentração no interior do grão, representado na metade de um corte longitudinal que passa pelo eixo do cilindro, considerando-se simetria radial. A escala, ao lado direito, informa a concentração adimensional $\left(C_{A} / C_{A}\right)$, que neste caso varia de 0,7 a 1,0 . Para os parâmetros utilizados, considerando uma reação endotérmica, pois $\beta<0$, o fator de efetividade calculado, de 0,459 , indica uma forte limitação difusional.

Catalisador em Forma de Cilindro vazado: Para este catalisador os resultados estão apresentados na Figura 4. Podem-se verificar perfeitamente as diferenças na concentração ao longo do grão. Para os valores de $\varphi, \beta$ e $\gamma$ escolhidos, considerando uma reação exotérmica, há forte limitação difusional à transferência de massa e também de calor resultando num valor de $\eta$ muito alto $(\eta$ $=122,9)$, uma vez que com o aumento da temperatura do grão, as taxas de reações verificadas tornam-se muito altas. No entanto, a reação fica praticamente restrita à superfície externa.

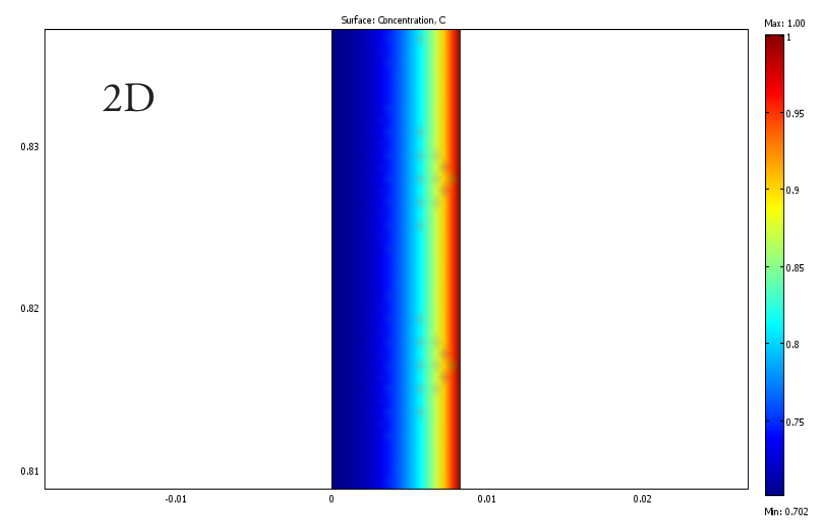

Figura 3 - Perfil de concentração do reagente no interior de um catalisador em forma de cilindro infinito, para $\gamma=20,0, \beta=-0,2$ (reação endotérmica) e $\varphi=1$. $\eta=0,459$ 


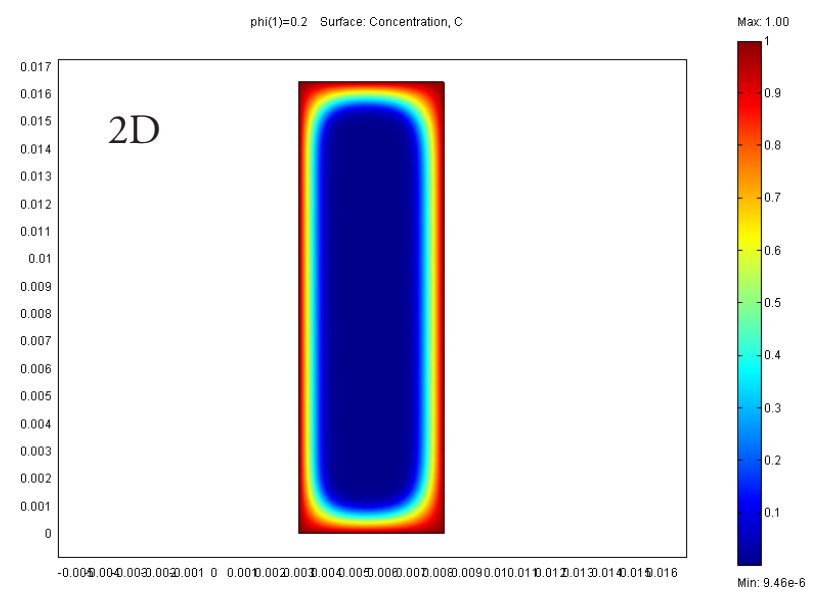

Figura 4 - Perfil de concentração do reagente ao longo do catalisador em forma de um cilindro vazado no meio, usando $\gamma=40, \beta=0,3$ (reação exotérmica) e $\varphi=0,2 \cdot \eta=122,9$

\subsection{RESULTADOS EM GRÁFICOS 3D}

Catalisador em Forma de Cilindro Fechado Finito: Como dito anteriormente os resultados expressos num gráfico 3D permitem melhor visualização dos fenômenos ocorridos, dos gradientes de concentração e temperatura e da própria geometria do grão. A Figura 5 apresenta o perfil de concentração para $\gamma=10, \beta=0$ e $\varphi=2$, sendo possível verificar as diferenças na concentração ao longo do grão catalítico, que variam entre 0,3 , no interior, e 1,0 na superfície externa. $O$ fator de efetividade calculado $(\eta)$ é de 0,242 .

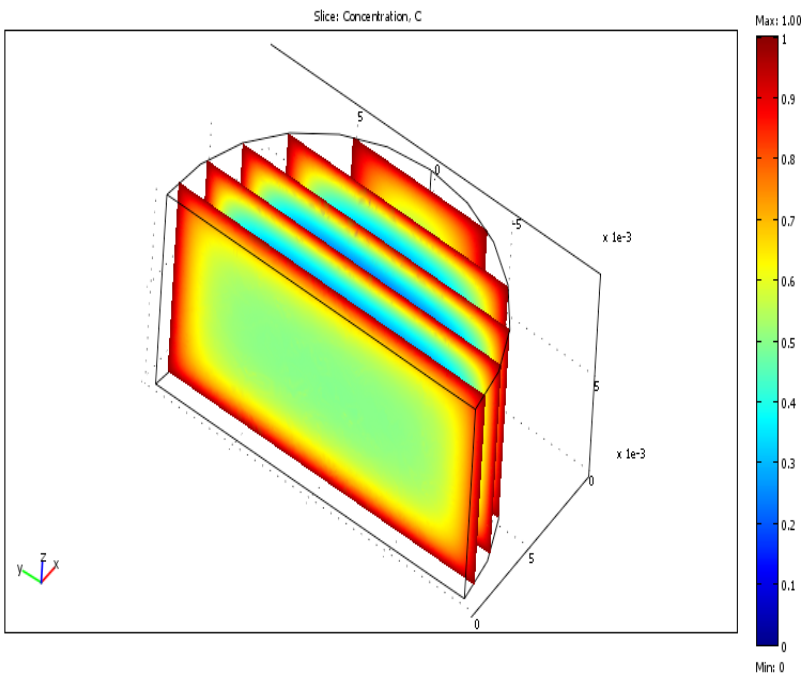

Figura 5 - Perfil de concentração do reagente ao longo do grão catalítico em forma de um cilindro fechado finito, usando $\gamma=10, \beta=0$ е $\varphi$ $=2$. $\eta=0,242$
Pela Figura 6, verifica-se que aumentando o Módulo de Thiele $(\varphi)$ para 4, fator relacionado ao tamanho do grão, para o mesmo catalisador, ocorre um aumento proeminente no gradiente de concentração ao longo do grão. Neste caso, não se pode desprezar os fluxos axiais. As diferenças nas tonalidades indicam que as concentrações dos reagentes, expresso pela razão $C_{A} / C_{A s}$ podem chegar a valores entre 0,3 e 0,4 na região bem próxima à superfície do grão, chegando praticamente a zero no centro, denotando forte limitação difusional interna. $\mathrm{O}$ fator de efetividade cai para 0,142.

Fazendo-se as variações dos parâmetros $\gamma$, $\beta$ e $\varphi$, o programa funciona como um laboratório virtual em que o usuário pode realizar experimentos, estudar a influência dos diversos parâmetros reacionais, avaliar seus resultados e assim consolidar conceitos.

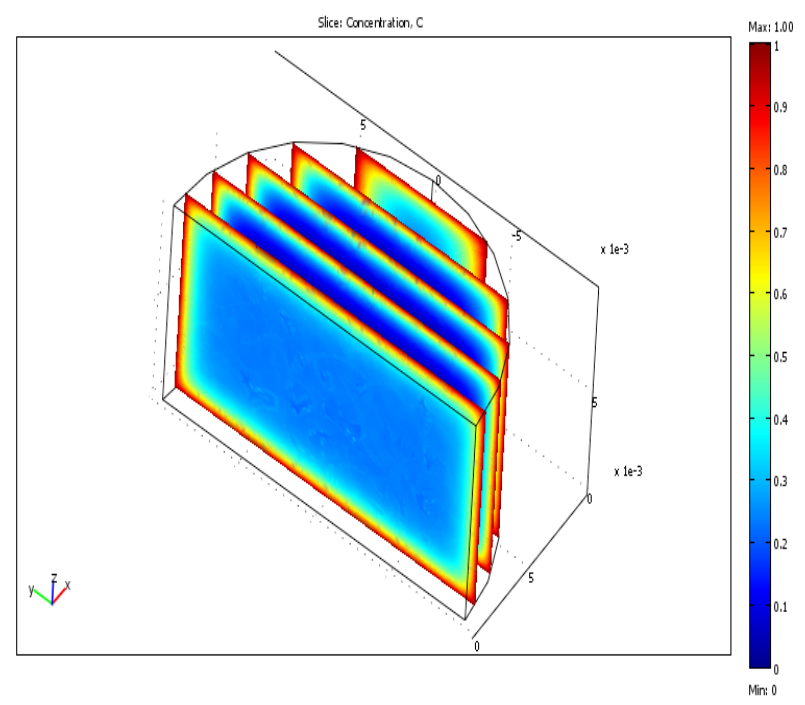

Figura 6 - Perfil de concentração do reagente ao longo do grão catalítico em forma de um cilindro fechado finito, usando $\gamma=10, \beta=0$ e $\varphi$

$$
=4 . \eta=0,142
$$

Catalisador em Forma de Cilindro vazado: As Figura 7 e 8 apresentam_os resultados expressos num gráfico $3 \mathrm{D}$, para grãos catalíticos vazados. Verifica-se que para $\gamma=10, \beta=0,2$ e $\varphi=0,5$, ocorrem limitações difusionais internas à transferência de massa de reagentes. No entanto, verifica-se que a área exposta à concentração de reagentes no grão vazado é bem maior do que para o grão na forma de um cilindro fechado. Isto indica que os catalisadores vazados permitem uma menor limitação difusional justificando sua utilização comercial.

Através da Figura 8, para condições mais drásticas de limitação difusional, $\operatorname{com} \gamma=40$, 
reação fortemente exotérmica, verifica-se que praticamente não há difusão de reagentes no interior do grão catalítico, com $C_{A} / C_{A s}=0 \mathrm{em}$ região bem próxima da superfície e em todo o interior do grão catalítico até o centro. Neste caso, observa-se um aumento no fator de efetividade chegando a um valor de 5,022, indicativo de uma forte limitação à transferência de calor interna. Por ser uma reação exotérmica, ocorre um aumento de temperatura no interior do grão, que pode provocar a desativação do catalisador por sinterização.

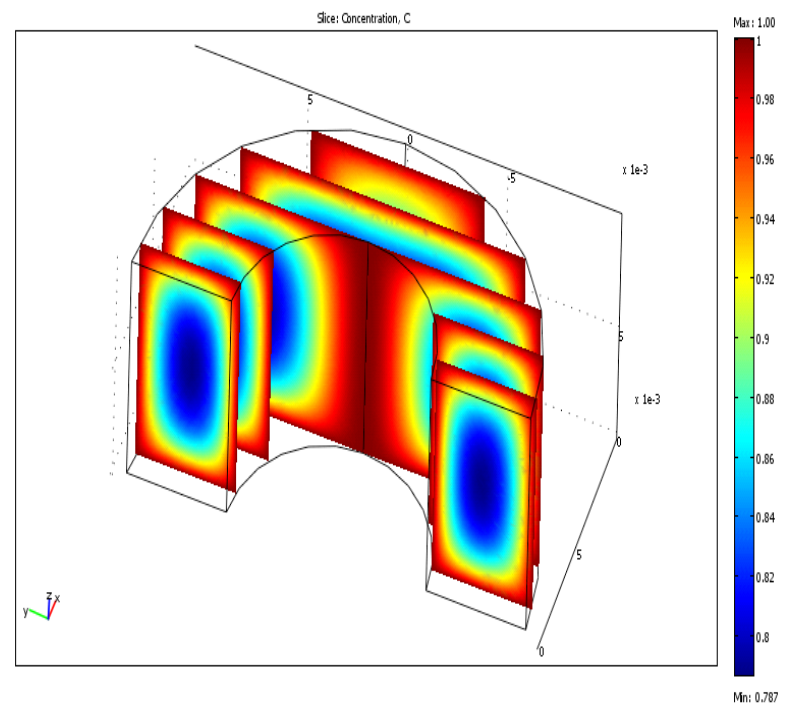

Figura 7 - Perfil de concentração do reagente ao longo do catalisador em forma de um cilindro vazado, para $\gamma=10, \beta=0,2$ e $\varphi=0,5$. $\eta=0,431$

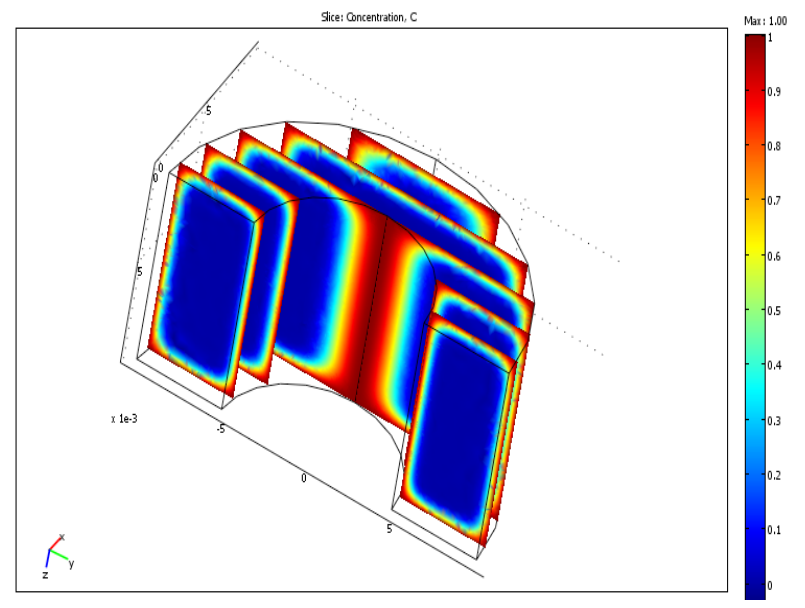

Figura 8 - Perfil de concentração ao longo do catalisador em forma de um cilindro vazado para $\gamma=40, \beta=0,2$ e $\varphi=0,5 . \eta=5,022$

A Figura 9 apresenta o perfil de temperatura para reaçôes exotérmicas sujeitas a grandes limi- tações difusionais internas, em que um aumento considerável na temperatura do interior do grão é observado (no centro $T / T_{S}=1,3$ ). Para um areação que ocorra a $700-800^{\circ} \mathrm{C}$, este aumento pode representar uma temperatura interna de 910$1040^{\circ} \mathrm{C}$.

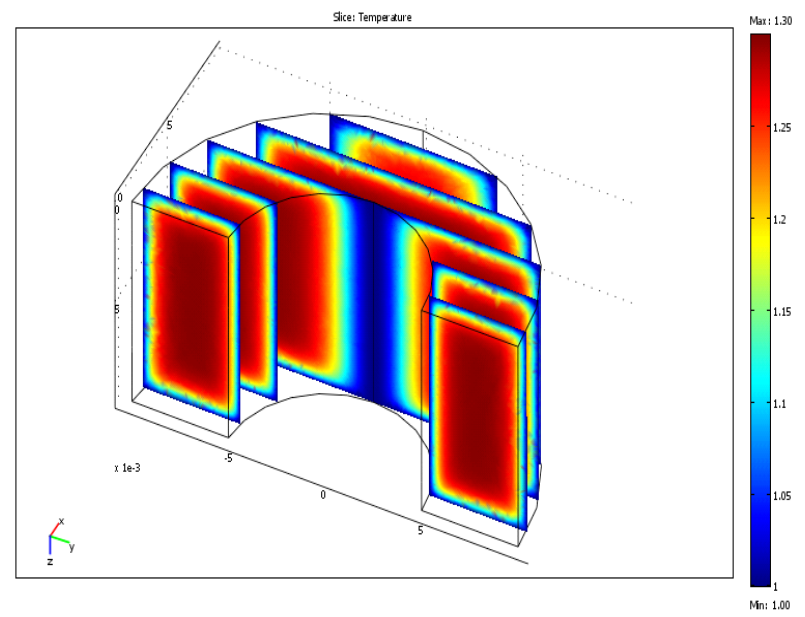

Figura 9 - Perfil de temperatura do reagente ao longo do catalisador em forma de um cilindro vazado, para $\boldsymbol{\gamma}=40, \boldsymbol{\beta}=\mathbf{0 , 3}$ е $\boldsymbol{\varphi}=\mathbf{1} \boldsymbol{\eta}=7,195$

Catalisadores em Forma de Cilindro com Quatro Furos e com Sete Furos: Especificamente para a reforma a vapor do metano, são comumente utilizados catalisadores contendo vários furos e reentrâncias para aumentar-se a área da superfície externa e assim a atividade catalítica. Nas condições usuais esperam-se valores de $\gamma$ entre 11 e 15 e valores de $\beta$ negativos, por ser uma reação endotérmica. Os perfis de concentração para esses tipos de catalisadores podem ser vistos através das Figuras 10 e 11, para os catalisadores de 4 furos e através das Figuras 12 e 13 para os de 7 furos, respectivamente.

Utilizando-se um valor típico para $\gamma=11,7$ e valores de $\beta=-0,1$ e $\varphi=2$, compatíveis com o processo industrial, observam-se grandes variações no perfil de concentrações para o catalisador de 4 furos. A Figura 11 apresenta um corte transversal no grão catalítico, para as mesmas condições apresentadas na Figura 10, para comparação, permitindo visualizar melhor os gradientes de concentração internos.

Comparando-se os resultados anteriores com os do catalisador de 7 furos, para os mesmos valores de $\varphi$, $\beta$ e $\gamma$, observa-se uma diminuição nas limitações difusionais, facilmente perceptíveis pelas diferenças nas tonalidades. A maior área 
superficial externa permite um maior acesso dos reagentes aos sítios de $\mathrm{Ni}$ dispersos na superfície, aumentando a atividade catalítica.

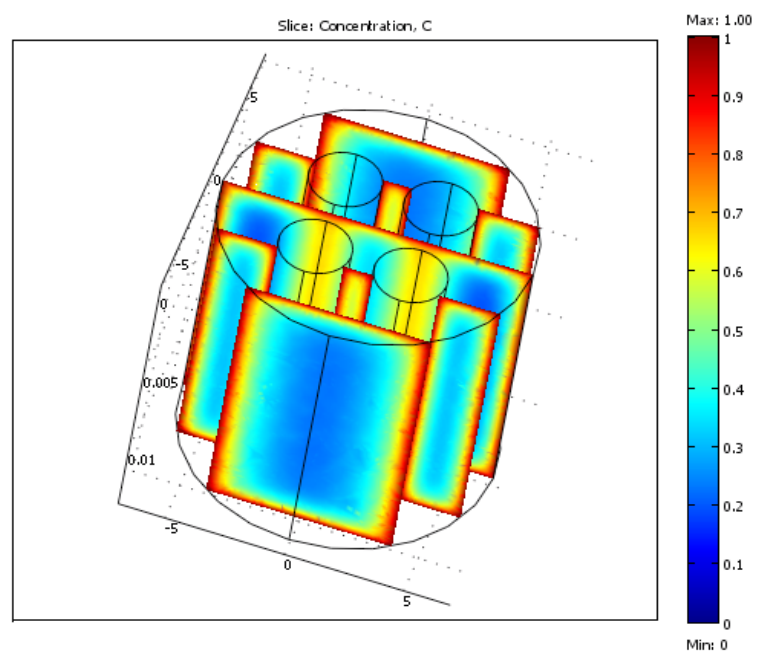

Figura 10 - Perfil de concentração do reagente ao longo do catalisador em forma de cilindro com quatro furos. $\gamma=11,7, \beta=-0,1$ e $\varphi=2$

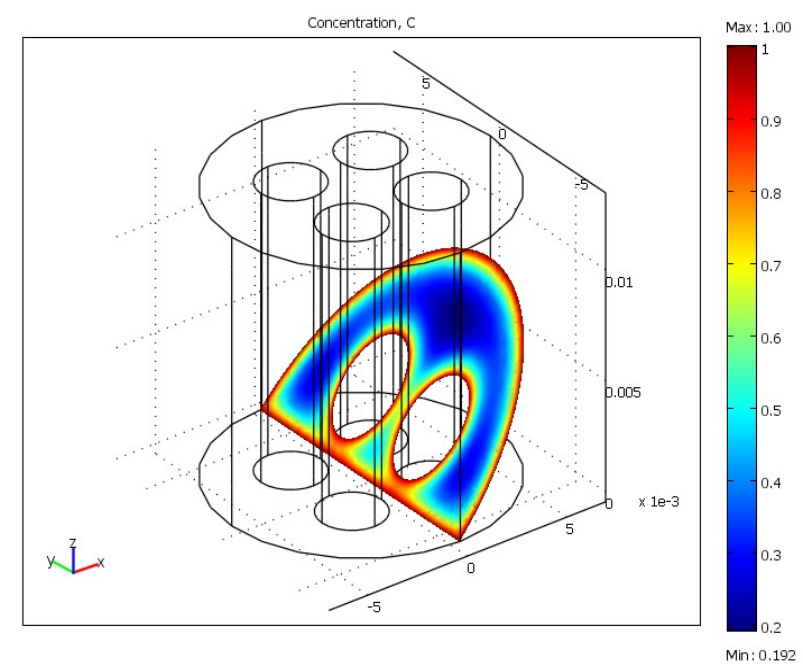

Figura 11 - Perfil de concentração do reagente ao longo do catalisador em forma de cilindro com quatro furos. $\gamma=11,7, \beta=-0,1$ e $\varphi=2$

Um corte transversal, apresentado na Figura 13, permite comparar o gradiente de concentração do catalisador de 7 furos com o de 4 furos, apresentado na Figura 11. Há muito menos área inacessível, para o de 7 furos do que para o de 4 furos. Isto explica a utilização em escala industrial de grãos catalíticos com vários furos e outras geometrias que permitam maior exposição da superfície ativa através de uma maior área externa.

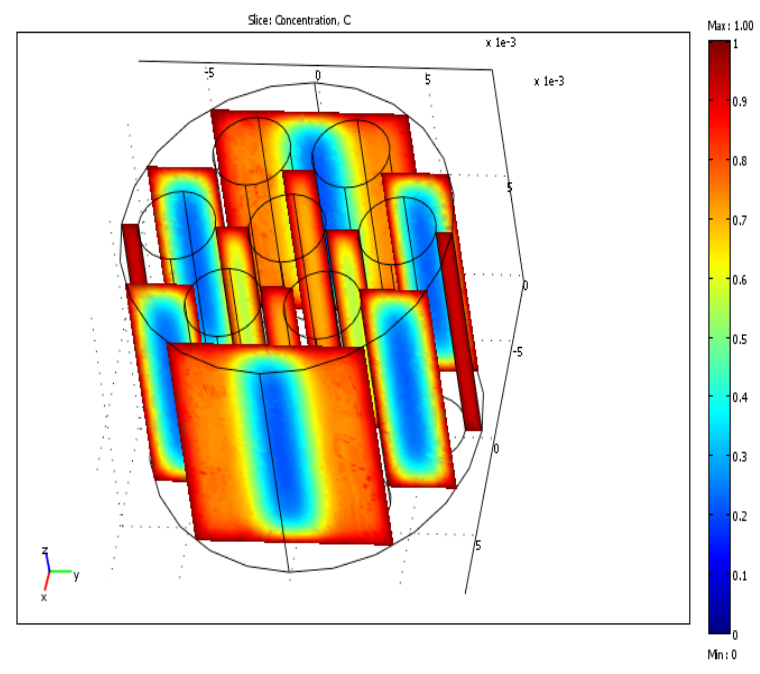

Figura 12 - Perfil de concentração do reagente ao longo do catalisador em forma de cilindro com sete furos, para $\gamma=11,7, \beta=-0,1$ e $\varphi=2$

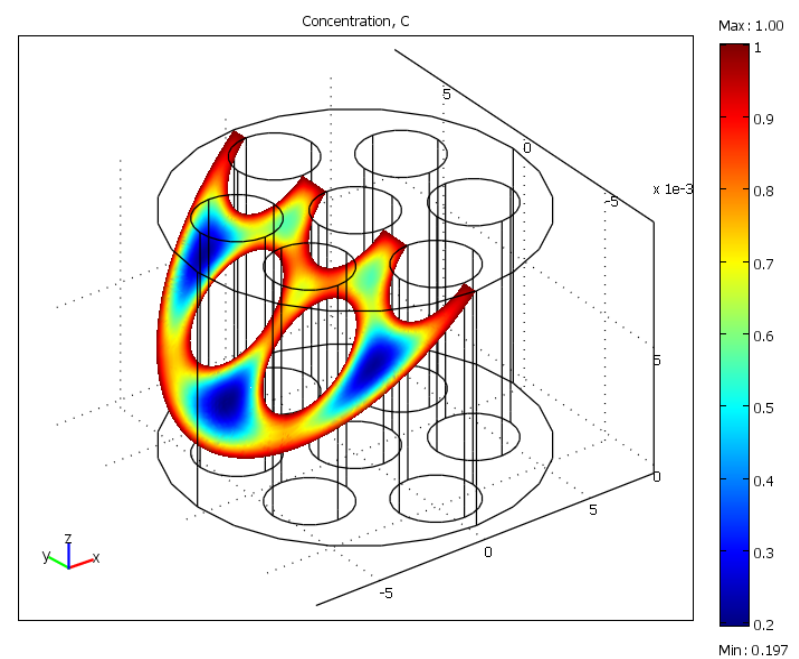

Figura 13 - Perfil de concentração do reagente ao longo do catalisador em forma de cilindro com sete furos. $\gamma=11,7, \beta=-0,1$ e $\varphi=2$

\subsection{COMPARAÇÃO ENTRE OS SOFTWARES}

Para permitir uma comparação entre os resultados usando os softwares MAPLE e COMSOL, a Figura 14 apresenta o gráfico contendo os valores de $\eta$ para os cilindros maciços e vazados infinitos, calculados a partir de ambos os softwares, o primeiro a partir de uma solução analítica obtida no MAPLE e o segundo utilizando métodos numéricos do COMSOL. Verifica-se que os resultados são concordantes e as curvas praticamente coincidentes.

Entretanto, para geometrias mais complexas não se pode utilizar o MAPLE. O COMSOL 
resolve o problema numericamente pelo método dos elementos finitos. Além disso, por ser um software CFD, permite a visualização do fenômeno envolvido

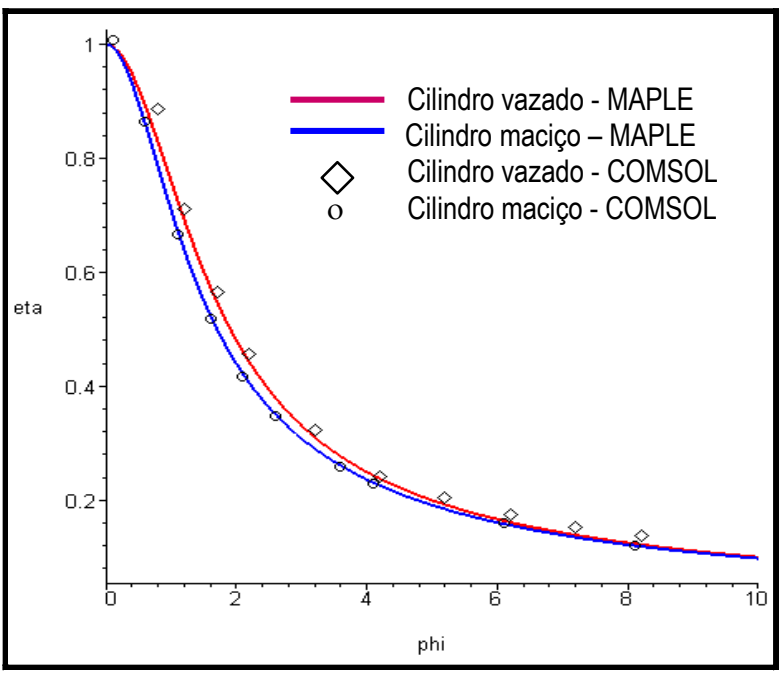

Figura 14 - Fator de efetividade em função do módulo de Thiele para grãos em forma de cilindro maciço e vazado infinitos calculados pelo MAPLE e pelo COMSOL $(\boldsymbol{\beta}=\mathbf{0})$

\section{CONCLUSÕES}

Os resultados indicam a importância da utilização do software COMSOL na solução de problemas complexos, em especial, nas reaçôes químicas catalíticas heterogêneas, que utilizam catalisadores sólidos, sujeitas às limitações difusionais internas à transferência de calor e massa, como a que ocorre na reforma a vapor do metano. Puderam-se utilizar diversas geometrias para os catalisadores, tais como os empregados industrialmente, e avaliar-se os gradientes de concentração e temperatura, calculando-se também os fatores de efetividade envolvidos. Neste contexto, o estudo de modelagem realizado permitiu simular e reproduzir adequadamente o comportamento desses sistemas catalíticos, permitindo testar e comparar a influência da forma dos diferentes catalisadores, apresentando resultados que podem ser utilizados como ferramentas preditivas para o projeto de sistemas reacionais catalíticos heterogêneos. Para esta reação em particular, os resultados indicam que um aumento do número de furos internos nos grãos catalíticos permite uma maior difusão dos reagentes. No entanto, deve-se considerar os problemas relativos a resistência mecânica dos grãos, sujeitos a altas temperaturas.
Do ponto de vista didático, a aplicação dessa abordagem possibilita uma melhor compreensão dos princípios básicos pelo estudante, ampliando o seu entendimento do conteúdo teórico. A possibilidade de testar suas próprias idéias, reformular conceitos e verificar se os resultados correspondem à realidade, desenvolve a capacidade crítica permitindo maior agregação de conhecimento e maior independência na sua formação acadêmica.

\section{REFERÊNCIAS}

ABEL, M. L.; BRASELTON, J. P. (1999). MAPLE V by Example New York: Academic Press.

CALDAS, F. V., SILVA, R. R. C. M. ROCHA, A. A. (2005). Ensinando Fenômenos de Transporte em um Ambiente Computacional Interativo Usando o Maple. In: XI Encontro de Educação em Engenharia, Penedo, RJ.

CALDAS, F. V., SILVA, R. R. C. M. ROCHA, A. A. (2009). Modelagem de problemas de reação em catalisadores porosos sujeitos à limitação difusional interna de calor e massa utilizando um ambiente computacional interativo utilizando o MAPLE. Revista ENGEVISTA, vol.11 , n 2, 117-126.

COMSOL Multiphysics ${ }^{\circledR}$. Modeling Guide, (2007). COMSOL AB.

CREMASCO, M. A. (2004). Fundamentos de Transferência de Massa, Editora da Unicamp.

FIGUEIREDO, J. I.; RIBEIRO, F. R. (1989). Catálise Heterogênea. Lisboa: Fundação Calouste Gulbenkian.

FOGLER, H. S. (2002). Elementos de Engenharia das Reações Químicas. Ed. Livros Técnicos e Científicos, Rio de Janeiro, RJ. Traduzido por Flávio Faria de Moraes e Luismar Marques Porto, da 3a ed. em inglês (1999) Elements of Chemical Reaction

FROMENT, G.F. BISCHOFF, K.B. (1979). Chemical Reactor Analysis \& Design. John Wiley $\&$ Sons, New York.

HECK, A. (1993). Introduction to Maple New York: Springer-Verlag. 
INCROPERA, F. P.; et al. (2003). Fundamentos de transferência de calor e de massa: LTC-Livros técnicos científicos editora, Rio de Janeiro.

MAYMO, J. A.; SMITH, J. M. (1966). Catalytic Oxidation of Hydrogen - Intrapellet Heat and Mass Transfer. A.I.Ch.E. Journal, vol. 12, no 5, 845-854.

MEADE, D. B.; HARAN, B. S.; WHITE, R. E. (1996). The Shooting Technique for the Solution of Two-Point Boundary Value Problems. Mapletech Computer Algebra, vol. 3, no 1. South Carolina: Waterloo Maple Inc.

NIJEMEISLAND, M., DIXON, A. G. E STITT, (2004) E. H. Chemical Eng. Science, 59, 51855191.

SATERFIELD, C. N. (1991). Heterogeneous Catalysis in Industrial Practice, $2^{\text {nd }}$ edition. New York. McGraw-Hill.
SILY, P. D.; CALDAS, F. V., ROCHA, A. A., SILVA, R. R. C. M. (2006) A Engenharia Química na Era Digital - Aplicação na Análise de Catalisadores Porosos com Limitação Difusional Interna de Calor e Massa. XVI Congresso Brasileiro de Engenharia Quimica, Anais em CDROM, Curitiba.

TWIGG, M. V. (1980) Catalyst Handbook, 2nd edition, edited by Martyn V. Twigg, pp. 225.

WEI, J., IGLESIA, E. (2004) Journal of Catalysis, 224, 370-383.

WEISZ, P. B.; HICKS, J. S. (1962). The Behaviour of Porous Catalyst Particles in View of Internal Mass and Heat Diffusion Effects. Chemical Engineering Science, vol. 17, 265-275. London: Pergamon Press Ltd. 